\title{
Discussion on LED Position in LED Total Luminous Flux Device
}

\author{
Peng $\mathrm{Xu}^{1}$, Xinye $\mathrm{Wu}^{2}$, Hailong $\mathrm{Bao}^{1}$, Yiping $\mathrm{Ji}^{1}$ and Wei Liao ${ }^{1}$ \\ ${ }^{1}$ State Grid Electric Power Research Institute of Shanghai Electric Power Corporation, Shanghai, 200437, China; \\ ${ }^{2}$ State Grid Shanghai Electric Power Corporation, Shanghai, 200122, China
}

\begin{abstract}
The development, production and application of Light Emitting Diodes (LEDs) have increased tremendously in the last ten years. All the special and useful properties of the LEDs are accompanied by their commensurate problems in measuring the light output. The spectrum of LED is quite different from the conventional incandescent source. In this paper we will examine the location of the LED in the integrating sphere when measuring the total flux output of the light source. We set up a mode of integrating sphere and a narrow bean light source by a photic design software. The conclusion from theoretical derivation and software simulation shows that the tested LED can be placed on the wall of the integrating sphere, and the wall location is much more suitable for LED rather than center position.
\end{abstract}

Keywords-LED; integrating sphere; flux

\section{INTRODUCTION}

The semiconductor technology has already triggered a microelectronics revolution and now triggering a new industrial revolution - the illumination revolution. The features of LED make it very useful in many fields such as display and special illumination. But because of its theoretical high light effect, one of its most potential great application prospects is the general illumination market.

The difficulty of the light source test is the luminous flux test. Compared with the traditional light source, although the International Lighting Commission (CIE) and the United States, Canada and other countries of the measurement research institutions have put forward the LED luminous flux test method, there is no simple test method which can be similar to the traditional light source flux measurement method due to the particularity of LED.

LED luminous flux test methods now available has several problems, including:

- The use of photovoltaic detector cannot match the LED visual function $\mathrm{V}(\lambda)$ in all the spectral point exactly, especially in the blue and red band;

- If the light source is placed in the integrating sphere or the screen is blocked when using traditional methods, the screen, the requirements of the integrating sphere theory will not be satisfied and resulting in test principle error since the test LED integrating sphere is generally small;

- Placing on the surface will make the standard lights which emit light to four side not available and the use of LED standard lights will cause a problem of cannot achieving spectral calibration.

These problems have caused different opinions on the LED luminous flux test and thus have affected the assessment of LED performance. It is not conducive to the development of LED industry. The main concern and discussion of this paper is: the reasonable position of the LED in the measurement of LED total luminous flux using the integrating sphere, i.e. whether the LED can be placed in the integral sphere wall instead of the center of the sphere.

\section{The MEASUREMENT PRINCIPLE OF TotAl LED LUMINOUS FLUX}

There are two methods to measure the total LED luminous flux: absolute measurement and relative measurement. Relative measurement is simpler than absolute measurement with less equipment investment.

The integrating sphere for relative measurement of the luminous flux is a hollow spherical shell, which has its inner wall covered with a smooth white diffusing layer. A window is opened on the wall of the sphere and the light source is placed inside the sphere. The light emitted by the light source is reflected by the inner wall and illuminated on the window receiver. The signal obtained by the receiver is proportional to the light flux of the light source.

In the total luminous flux method measured by integrating sphere, it is assumed that:

- The inner wall of the integral sphere is a uniform ideal diffuse reflection layer, following the Lambertian law.

- The diffuse coefficients of the points in the sphere are equal.

- The white pigment on the inner wall of the integrating sphere has the same diffusing coefficient for the light of various wavelengths emitted by the light source, that is, the diffusion of the spherical wall is neutral.

- The diameter of the sphere is the same, and there is no other debris outside the bulb. 


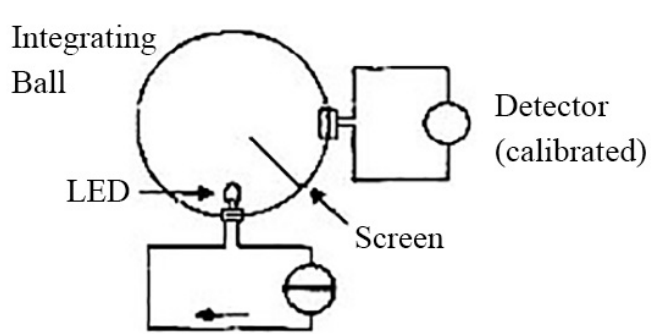

FIGURE I. ILLUSTRATION OF THE TOTAL LUMINOUS LED FLUX MEASUREMENT WITH INTEGRATING SPHERE

Light up the standard light in the position of the light source inside the integrating sphere. A signal is obtained by the receiver in the light window. Remove the standard light and light up the LED and another signal is obtained. The total luminous flux of LED is calculated as follows:

$$
\Phi=k \frac{i}{i_{s}} \Phi_{s}
$$

Where $k$ is the color correction factor and $\Phi s$ is the standard light flux.

\section{RESONABLE POSITION OF LED IN THE INTEGRATING SPHERE}

It is important to note here that in the integrating sphere measurement of conventional light sources, the light source is often placed in the center of the integrating sphere. However, LED is a new type of light source and has a certain degree of particularity:

- The total luminous flux output of the LED decreases rapidly when its own temperature rises. Working LED requires a good cooling in order to ensure the stability of its luminous flux output. But the confined space of the integrating sphere cannot meet the needs of its cooling.

- The volume of LED is small but the luminous flux caused by the light device in the integrating sphere cannot be ignored.

If the measurement mode of conventional light source in the center of integrating sphere is still used, the above LED characteristics will inevitably bring errors to the measurement results. However, if the test position of the light source is placed on the wall of the integrating sphere, the above problems will be solved.

The following parts of this section validates whether the illumination of the light source on the wall obtained by the receiver is proportional to its total luminous flux.

\section{A. Theoretical Derivation}

According to the integrating sphere principle, when the light source is placed in any position within the sphere, the light window position illumination is:

$$
E=E_{1}+\frac{\Phi}{4 \pi r^{2}} \cdot \frac{\rho}{1-\rho}
$$

Where $E$ is the illumination on the light window in units of 1x. $E_{1}$ is the direct illumination of light emitted by the light source on the light window, in units of $1 \mathrm{x} . \Phi$ is the total luminous flux of the light source, unit $\mathrm{lm} . r$ is the radius of the integrating sphere in units of $\mathrm{m}$. $\rho$ is the diffuse rate of the inner wall of the integrating sphere.

If a block screen is placed in the sphere to block the light from the light source, the illumination on the position of the light window is:

$$
E=\frac{\Phi}{4 \pi r^{2}} \cdot \frac{\rho}{1-\rho}
$$

The signal obtained by the receiver is proportional to the total luminous flux of the light source.

It can be seen from the above equation that regardless of the position of the light source in the integrating sphere, the signal obtained by the receiver is the same. That is, the signal value obtained by the receiver independent of the light source position. .

\section{B. Software Modeling Validation}

An integrating sphere model was established by optical simulation software.

The model is shown in Figure 2. A plane light source $(1 \mathrm{~mm}$ $\times 1 \mathrm{~mm}$ ) of the unidirectional beam was placed at the inner wall of the sphere, the reflectance of the inner wall was $99 \%$ and the diameter of the sphere was $300 \mathrm{~mm}$. The light window and the light source were placed perpendicular to each other in the same plane.

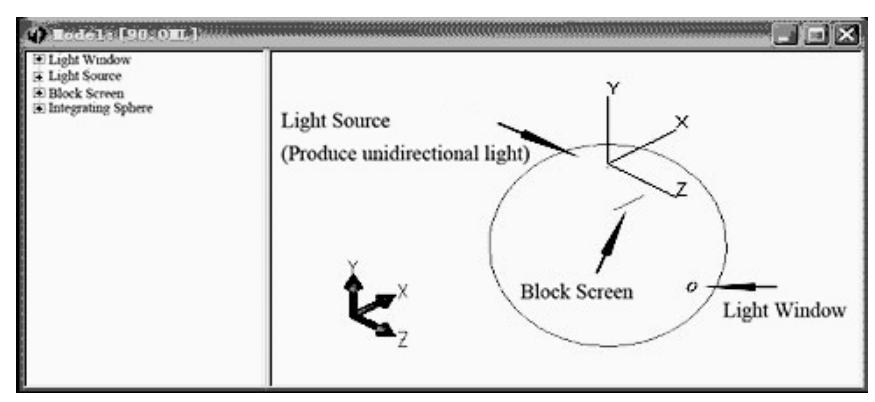

FIGURE II. SOFTWARE MODELING OF INTEGRATING SPHERE MEASUREMENT LED TOTAL LUMINOUS FLUX DIAGRAM

In the Y-Z plane, the light angle of the light source was defined from 0 degree and the light tracing was performed on the 15,000 rays per 10 degree with the Monte Carlo method to obtain the luminous flux received on the optical window. 


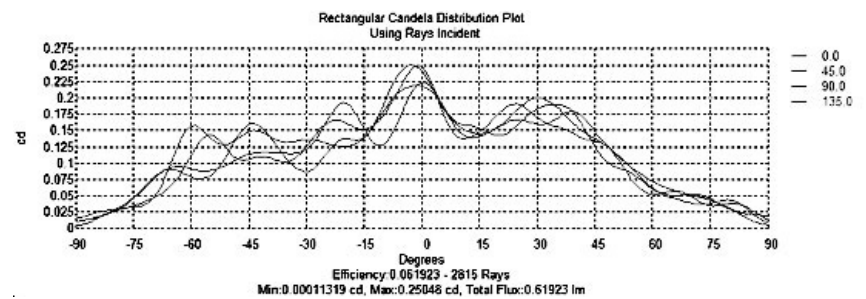

FIGURE III. LUMINOUS FLUX AT LIGHT WINDOW

Thus, the luminous flux at the light window in different light directions and with different light source positions was obtained. And compared it with the theoretical calculated value ( $\Phi=0.61776 \mathrm{~lm}$ ) of the luminous flux at the light window:

TABLE I. COMPARISON OF LUMINOUS FLUX VALUES IN DIFFERENT LIGHT DIRECTION WITH THEORETICAL VALUES

\begin{tabular}{|c|c|c|c|c|c|}
\hline $\begin{array}{c}\text { Angle } \\
\text { (degree) }\end{array}$ & $\mathbf{0}$ & $\mathbf{1 0}$ & $\mathbf{2 0}$ & $\mathbf{3 0}$ & $\mathbf{4 0}$ \\
\hline $\begin{array}{c}\text { Comparison with } \\
\text { theoretical values (\%) }\end{array}$ & -4.42 & -4.72 & 0.21 & -5.20 & 0.35 \\
\hline $\begin{array}{c}\text { Angle } \\
\text { (degree) }\end{array}$ & $\mathbf{5 0}$ & $\mathbf{6 0}$ & $\mathbf{7 0}$ & $\mathbf{8 0}$ & $\mathbf{9 0}$ \\
\hline $\begin{array}{c}\text { Comparison with } \\
\text { theoretical values (\%) }\end{array}$ & -7.00 & 0.70 & 2.88 & 0.04 & -3.24 \\
\hline $\begin{array}{c}\text { Angle } \\
\text { (degree) }\end{array}$ & $\mathbf{1 0 0}$ & $\mathbf{1 1 0}$ & $\mathbf{1 2 0}$ & $\mathbf{1 3 0}$ & $\mathbf{1 4 0}$ \\
\hline $\begin{array}{c}\text { Comparison with } \\
\text { theoretical values (\%) }\end{array}$ & -2.13 & -0.09 & 2.79 & -2.25 & -4.52 \\
\hline $\begin{array}{c}\text { Angle } \\
\text { (degree) }\end{array}$ & $\mathbf{1 5 0}$ & $\mathbf{1 6 0}$ & $\mathbf{1 7 0}$ & $\mathbf{1 8 0}$ & \\
\hline $\begin{array}{c}\text { Comparison with } \\
\text { theoretical values (\%) }\end{array}$ & -5.00 & -0.18 & -0.80 & -3.70 & \\
\hline
\end{tabular}

And then obtain the luminous flux on the light window from a point source with a certain light angle in different positions. And compared it with the theoretical calculated value ( $\Phi=0.61776 \mathrm{~lm}$ ) of the luminous flux at the light window:

Due to the limitation of computing capability, only 150,000 rays were subjected to Monte Carlo trace. Thus, there was a certain error between the simulation result and the theoretical calculation of the luminous flux at the light window. The error was in the range of $\pm 5 \%$. The cause of the error was inextricably linked to the limited number of traces. The cause of the error was inseparable from the limited number of tracing light. When the number of trace light increased, the luminous flux obtained at the light window would be closer to the theoretical calculation value. Therefore, it was reasonable to assume that placing the LED light source on the inner wall of the integrating sphere was feasible.
TABLE II. COMPARISON OF LUMINOUS FLUX VALUES IN DIFFERENT SOURCE POSITION WITH THEORETICAL VALUES

\begin{tabular}{|c|c|c|c|c|c|c|}
\hline \multirow{2}{*}{\multicolumn{2}{|c|}{$\begin{array}{c}\text { Angle } \\
\text { (degree) }\end{array}$}} & $\mathbf{0}$ & 45 & 90 & 135 & 180 \\
\hline & & \multicolumn{5}{|c|}{ Comparison with theoretical values (\%) } \\
\hline \multirow{20}{*}{$\begin{array}{c}\text { Distance } \\
\text { between the } \\
\text { light source } \\
\text { and the } \\
\text { wall of } \\
\text { integrating } \\
\text { sphere } \\
\text { (mm) }\end{array}$} & 1 & -2.90 & -5.59 & \multirow{20}{*}{-3.24} & -0.88 & -2.16 \\
\hline & 2 & -0.72 & -3.05 & & -1.73 & -1.80 \\
\hline & 3 & -0.48 & -3.91 & & -3.17 & -0.51 \\
\hline & 4 & -0.92 & -4.48 & & -0.14 & -1.99 \\
\hline & 5 & -0.91 & -0.52 & & -3.60 & 0.34 \\
\hline & 6 & 1.35 & -2.29 & & -1.72 & 1.56 \\
\hline & 7 & -3.67 & -4.37 & & -3.42 & -5.09 \\
\hline & 8 & -0.21 & 0.17 & & -2.89 & -0.86 \\
\hline & 9 & -0.42 & -0.97 & & -0.96 & -2.23 \\
\hline & 10 & -5.09 & -1.38 & & -0.76 & 1.41 \\
\hline & 11 & -5.13 & -4.20 & & -1.38 & -1.38 \\
\hline & 12 & -2.25 & -3.41 & & -5.01 & -6.09 \\
\hline & 13 & -3.10 & -2.42 & & -1.48 & -2.00 \\
\hline & 14 & 0.23 & -0.36 & & -0.13 & -5.71 \\
\hline & 15 & 0.69 & -2.10 & & 0.43 & 0.90 \\
\hline & 16 & 3.21 & -0.43 & & -1.50 & -2.87 \\
\hline & 17 & 3.32 & -3.97 & & -4.99 & -3.09 \\
\hline & 18 & -1.37 & 1.37 & & -2.83 & 1.36 \\
\hline & 19 & 0.51 & 0.78 & & -2.59 & -3.84 \\
\hline & 20 & -1.80 & 0.48 & & -5.85 & 0.91 \\
\hline
\end{tabular}

\section{Discussion}

a) The size of integrating sphere:

Placing the block screen in the integrating sphere undermined the hypothesis of ideal integrating sphere. In order to reduce the impact of the block screen on the measurement results as much as possible, the size of the integrating sphere should be correspondingly large. Refer to the LED measurement method of CIE (Commission Internationale de L'Eclairage), the diameter of the integrating sphere for LED total luminous flux measurement should be greater than $30 \mathrm{~cm}$. If a smaller integrating sphere (such as a diameter of $10 \mathrm{~cm}$ ) is used for measurement, an auxiliary LED should be introduced for self-absorption correction (the self-absorption correction coefficient is $\alpha$ ).

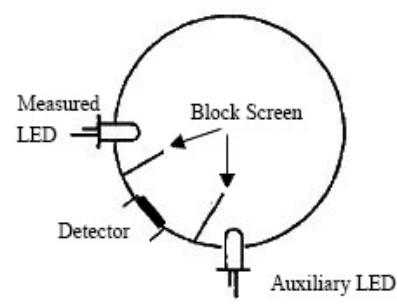

FIGURE IV. ILLUSTRATION OF USING AUXILIARY LED

$\alpha$ is calculated as in (4): 


$$
\alpha=\frac{\text { Illumination of the standard lamp }}{\text { Illumination of the measured lamp }} \text {. }
$$

\section{b) Standard light source:}

There are wide differences between LED and traditional thermal radiation source in volume, spectral distribution, light angle and other characteristics. In the LED luminous flux measurement with the integrating sphere, if tungsten wire is still used as a standard light source, a great deal of selfabsorption and relative spectral sensitivity errors will be introduced.

Therefore, the standard light source should be an LED source. The total luminous flux and light distribution curve of a standard LED can be determined by spectrophotometer in advance.

As a standard light source, its light distribution curve and spectral distribution needs to be the same as the LED under test. If it cannot fully meet the requirements, the difference of relative spectral sensitivity introduced by different spectral distributions can be corrected by (5):

$$
\alpha=\frac{\int_{0}^{\infty} S(\lambda) \cdot V(\lambda) d \lambda}{\int_{0}^{\infty} S_{0}(\lambda) \cdot V(\lambda) d \lambda} \cdot \frac{\int_{0}^{\infty} S(\lambda) \cdot W(\lambda) \cdot D(\lambda) \cdot \tau(\lambda) d \lambda}{\int_{0}^{\infty} S_{0}(\lambda) \cdot W(\lambda) \cdot D(\lambda) \cdot \tau(\lambda) d \lambda} .
$$

Where $S(\lambda)$ and $S_{0}(\lambda)$ are the spectral power distribution of the measured LED and the standard LED respectively. $\tau(\lambda)$ is the relative spectral transmittance of the window. $D(\lambda)$ is the relative spectral sensitivity of the detector. And $W(\lambda)$ can be calculated in (6):

$$
W(\lambda)=K \cdot \frac{\rho(\lambda)}{1-\rho(\lambda)}
$$

Where $\rho(\lambda)$ is the reflectivity of the inner surface of the integrating sphere and $K$ is an arbitrary constant.

\section{CONCLUSION}

In the measurement of total LED luminous flux, it is feasible and scientific to set the LED on the inner wall of the integrating sphere instead of the center of the LED in the relative method. This method not only avoids the decrease of luminous flux caused by the temperature rise when the LED is lighted, but also can satisfy the integrating sphere principle as far as possible by the utility model, so the self-absorption of the lighting device is reduced. For the narrow light distribution of LED light source, when the light source and the detector probe are placed vertically in the same plane, the block screen will no longer be needed because little light from LED can go through the probe directly. Thus, the principle of integrating sphere is further met and the LED flux measurement accuracy is improved.

\section{REFERENCES}

[1] C. Bao, "The measurement and calibration of light emitting diodes," in Physics, vol. 32, pp. 320-321, 2003.

[2] Z. Lu, Q. Fang, and L. Lu, "Self-absorption effect and its correction with integrating spheres to measuring LEDs luminous flux," in China Illuminating Engineering Journal, vol. 17, pp. 32, 2006.

[3] Z. Lu, "Several problems in LED luminous flux testing," in China Light \& Lighting, vol. 12, pp. 29-30, 2005.

[4] C. Bao, "Problems About Measurement of Superhigh Intensity LED," in Chinese Journal of Liquid Crystals and Displays, vol. 18, pp. 247, 2003.

[5] CIE, Measurement of LEDs, CIE127-1997

[6] A. A. Gaertner, "LED measurement issues," in Optronic Laboratories' website, pp. 11-13, 2002 